Document downloaded from:

http://hdl.handle.net/10251/179848

This paper must be cited as:

Wang, Y.; Sun, H.; Khalil, M.; Dong, W.; Gasulla Mestre, I.; Capmany Francoy, J.; Chen, LR. (2020). Multi-Cavity Optoelectronic Oscillators Based on an Integrated Array of Subwavelength Grating Waveguides. IEEE. 184-187. https://doi.org/10.23919/MWP48676.2020.9314495

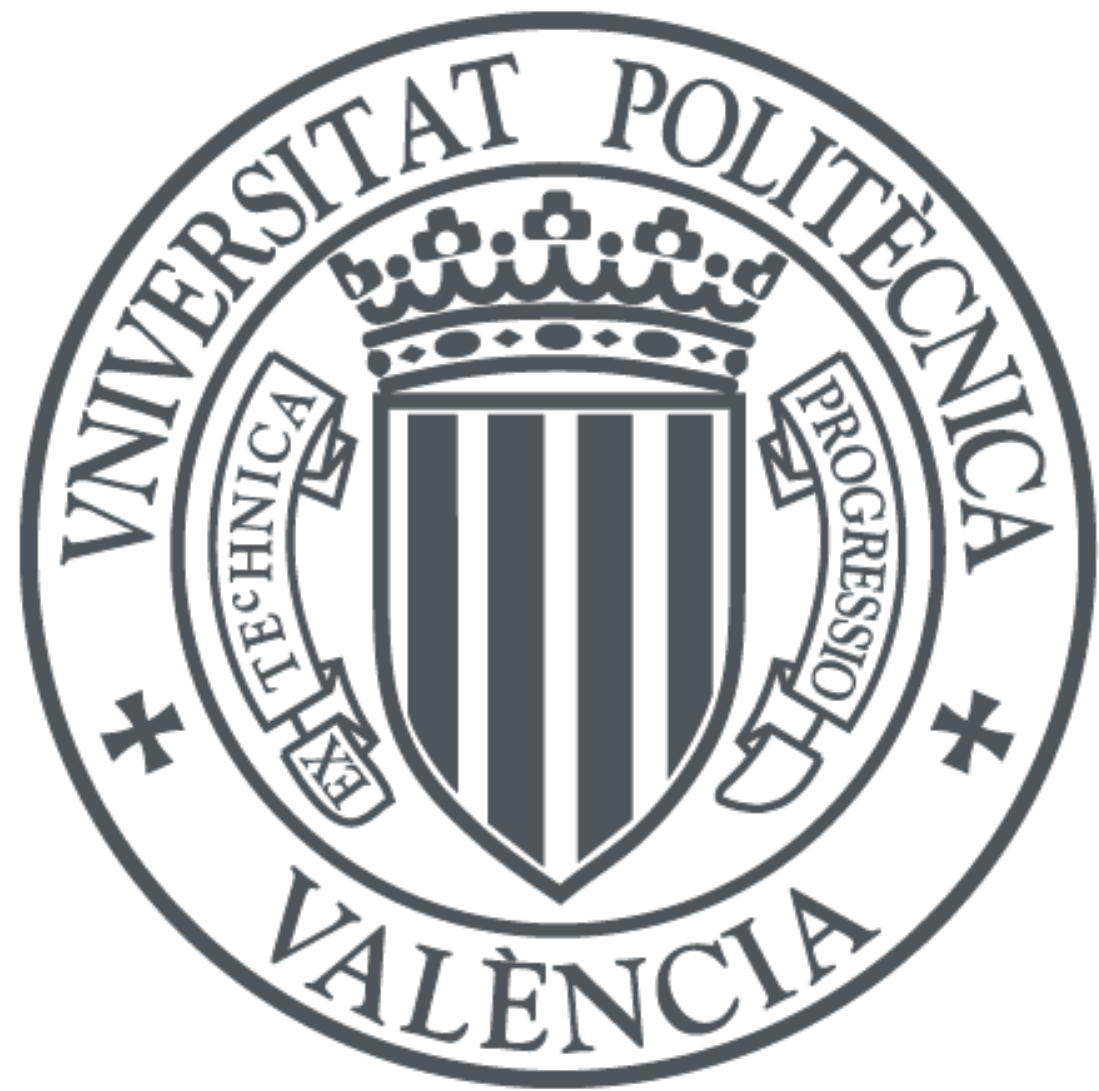

The final publication is available at

https://doi.org/10.23919/MWP48676.2020.9314495

Copyright IEEE

Additional Information 


\title{
Microwave Photonics Applications of On-chip Optical True Time Delay Lines Based on Subwavelength Grating Waveguides
}

\author{
Yue Wang, Hao Sun, Mostafa Khalil, Wei Dong, Ivana Gasulla, Senior Member, IEEE, \\ José Capmany, Fellow, IEEE, Fellow, OSA, \\ and Lawrence R. Chen, Senior Member, IEEE, Fellow, OSA
}

\begin{abstract}
An optical true time delay line (OTTDL) is a fundamental building block for signal processing applications in microwave photonics (MWP) and optical communications. Here, we experimentally demonstrate an index-variable OTTDL based on an array of forty subwavelength grating (SWG) waveguides on silicon-on-insulator (SOI). The SWG waveguides are arranged in a serpentine manner to increase the total length while maintaining a compact size; the array occupies a chip area of $\sim 6.5 \mathrm{~mm} \times 8.7$ $\mathrm{mm}=56.55 \mathrm{~mm}^{2}$. Each SWG waveguide in the array is $34 \mathrm{~mm}$ long, the average incremental delay between waveguides is $\sim 4.7$ ps, and the total delay between the first and last waveguides is 181.9 ps. We use numerical simulations with the measured time delays of the SWG waveguide-based OTTDL to demonstrate their implementation in various MWP applications. First, we show a microwave photonic filter (MPF) with discrete frequency tunability and reconfigurability. Second, we illustrate discrete tunability of the beamforming angle for a phased array antenna. Finally, we determine the oscillation spectra and phase noise of unbalanced dual-cavity optoelectronic oscillators (OEOs) and multi-cavity Vernier OEOs, as well as demonstrate the feasibility for discrete frequency tuning. The developed OTTDLs bring advantages in terms of compactness as well as operation versatility to a variety of microwave signal processing applications.
\end{abstract}

Index Terms-Silicon photonics, microwave photonics, microwave photonic filtering, optical beamforming, optoelectronic oscillators.

\section{INTRODUCTION}

$\mathrm{M}$ ICROWAVE photonics (MWP) is an interdisciplinary area that combines microwave and optical engineering and focuses on the use of photonic means to generate, distribute, and process microwave signals [1-4]. The strong interest in MWP lies in its numerous intrinsic advantages, such as broad operation bandwidth, strong immunity to electromagnetic interference, and no limitation due to the electronic bottleneck effect $[5,6]$. Recently, considerable

Submitted 21 July 2020. This work was supported in part by the Natural Sciences and Engineering Research Council of Canada, the China Scholarship Council, and the European Research Council under Consolidator Grant Project 724663

Y. Wang and D. Wei are with the State Key Laboratory on Integrated Optoelectronics, College of Electronic Science and Engineering, Jilin University, Changchun 130012, China. Y. Wang is also with the Department of progress has been directed on developing photonic technologies to realize MWP signal processing functions [7-10]. One such technology is an optical true time delay line (OTTDL), which is a fundamental building block for MWP discrete-time signal processing applications [11]. For instance, they can be applied to reconfigurable MWP filters, arbitrary waveform generation/shaping, multi-cavity optoelectronic oscillation, and optical beamforming in phased array antennas $[12,13]$. Various approaches exist to implement OTTDLs apart from switched variable-length single mode fiber (SMF) [14] configurations, including the exploitation of the optical wavelength diversity through passive dispersive elements, e.g., fiber Bragg gratings (FBGs) $[15,16]$ or exploiting the dispersion associated with a gain resonance, e.g., from stimulated Brillouin scattering [17, 18]. Characteristics of OTTDLs include large delay, small incremental delay steps for the discretely tunable delay, broad operating bandwidth, and low loss. It is not necessary for an OTTDL to possess all these features simultaneously as the requirements will depend on the specific application.

There are two general approaches for implementing an optical delay line (ODL) [10]: (1) varying the propagation length $(L)$ of the delay element (i.e., a length-variable delay line) $[19,20]$ and (2) varying the propagation group velocity $\left(v_{g}\right)$ (i.e., a wavelength-variable delay line) [21]. These two methods use fixed-length waveguides, optical fibers, or free space as the delay medium; they can also utilize resonance enhancements to go beyond the physical length of the delay medium. An ODL providing time delays for pulses/signals having the same optical carrier is one form of an OTTDL [10].

Recently, there has been significant interest in developing subwavelength grating (SWG) waveguide structures for highperformance photonic integrated circuits in silicon-on-insulator (SOI) $[22,23]$. An SWG waveguide comprises a periodic arrangement of two different materials, one with a refractive index that is higher than the other, with a period that is small enough to suppress the diffraction effects. The characteristics

Electrical and Computer Engineering, McGill University, Montreal, QC H3A 0E9 Canada.

H. Sun, M. Khalil, and L. R. Chen are with the Department of Electrical and Computer Engineering, McGill University, Montreal, QC H3A 0E9 Canada. (corresponding author: Lawrence R. Chen, email: lawrence.chen@mcgill.ca)

Ivana Gasulla and José Capmany are with ITEAM Research Institute, Universitat Politècnica de València, Valencia 46022, Spain. 


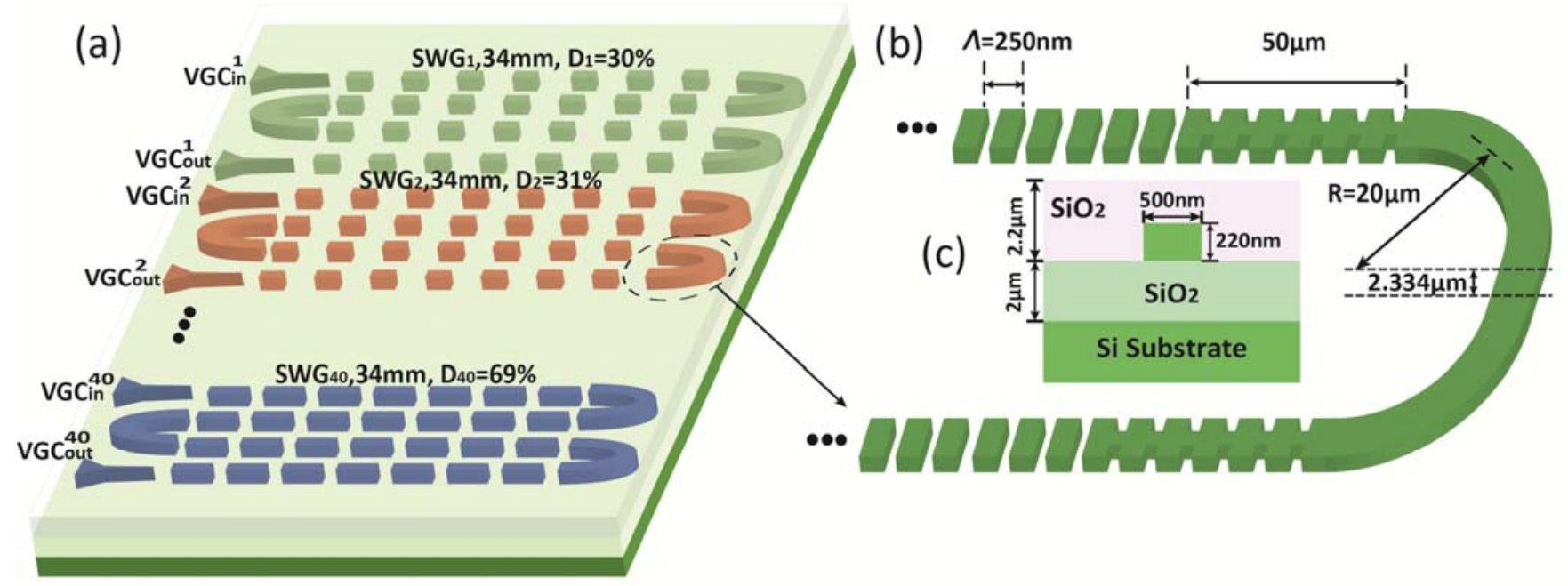

(d)

(e)
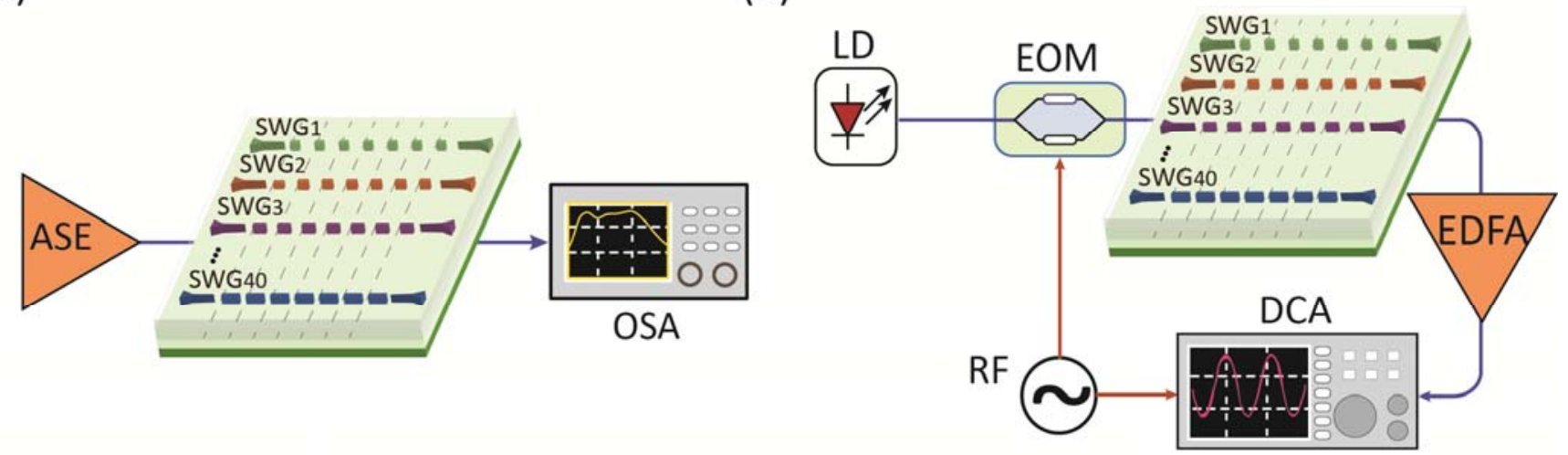

Fig. 1. Design and fabrication of SWG waveguide-based OTTDL and experimental characterization. (a) Schematic of the fabricated array of forty SWG waveguides in SOI (the different colors represent different duty cycles of the SWG waveguides). (b) Details of the bends. (c) The waveguide cross-section view. (d) Experimental setup of measuring power spectral response of the fabricated index-variable OTTDL. (e) Experimental time-of-flight measurement setup. ASE: amplified spontaneous emission source; OSA: optical spectrum analyzer; LD: laser diode; EOM: electro-optic intensity modulator; EDFA: erbium-doped optical fiber amplifier; DCA: digital communication analyzer; RF: RF generator.

of SWG waveguides, such as low loss and the flexibility to tailor the effective refractive index (through control of the duty cycle, defined as the ratio between the length of the high refractive index material to the period), can result in enhanced performance compared to conventional SOI nanowire waveguide-based devices [24]. Gasulla and Capmany exploited the parallelism of multicore fibers and proposed their use as a space sampled index-variable OTTDL for MWP applications [25]. Heterogeneous multicore fibers provide different propagation delays through each core by proper design of their physical dimensions and material doping concentrations: a differential time delay of a few $\mathrm{ps} / \mathrm{km}$ was achieved by tailoring an independent dispersion profile per core [26, 27]. Inspired by this approach, we proposed and demonstrated for the first time how an array of SWG waveguides can be used to implement an integrated version of heterogeneous multicore fibers as a sampled index-variable OTTDL [28]. In particular, the array involved four SWG waveguides of the same length but different propagation velocities, which were obtained by tailoring the effective index of each SWG waveguide through control of their duty cycles.

Here, we extend significantly our proof-of-concept and demonstrate an array of forty SWG waveguides to implement an index-variable OTTDL. We use numerical simulations in conjunction with the measured delays of our integrated SWG waveguide-based OTTDLs to demonstrate their applications in MWP. First, we show a microwave photonic filter (MPF) featuring discrete frequency tunability and reconfigurability: we calculate the filter responses of two-tap, three-tap, four-tap, and five-tap MPFs with different free spectral ranges (FSRs). Second, we determine the array factors for different phased array antennas. By proper choice of the waveguides to obtain a specific incremental time delay, we can realize discrete tuning of the beamforming angle. Moreover, when we fix the number of antenna elements, the beamforming angle can be tuned discretely without impacting the directionality of the beamforming patterns. Finally, we emulate the unbalanced dual-cavity optoelectronic oscillator (OEO) and multi-cavity Vernier OEO and demonstrate discrete tuning of the output frequency. 
(a)

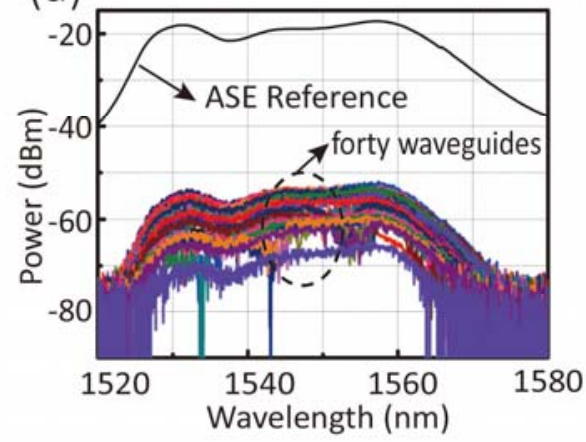

(b)

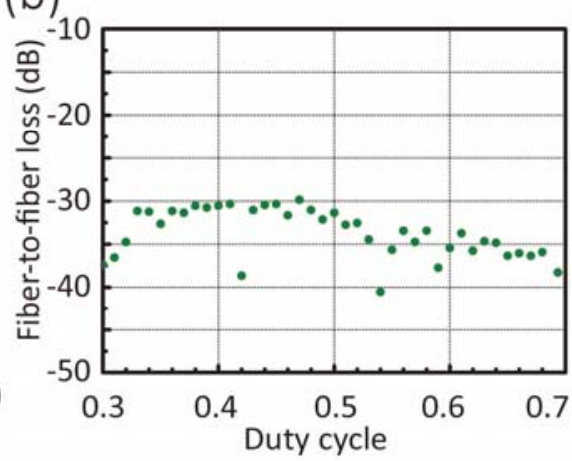

(c)

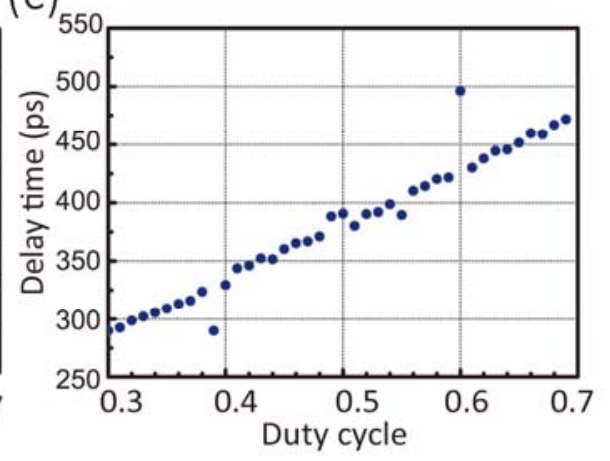

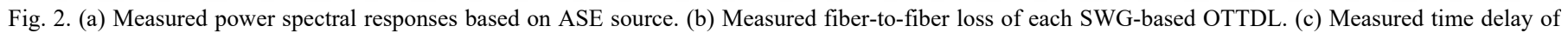
each OTTDL.

\section{Design AND CHARACTERIZATION OF THE SWG WAVEGUIDE-BASED OTTDLS}

An SWG comprises a periodic arrangement (where the period is $\Lambda$ ) of a medium with a higher refractive index (such as silicon) having a thickness $a$ embedded within a medium of lower refractive (such as silica). The effective index of SWG waveguides depends on the duty cycle $D$, and is defined as $D=a / \Lambda$, where $a$ is the length of the silicon segment in each period. An index-variable OTTDL generally involve waveguides of the same length but the propagation velocities are different. The group index of the SWG waveguides can be engineered to control the incremental time delay by choosing the duty cycles of each SWG waveguides. The group index of an SWG waveguide can be expressed as [28]:

$$
n_{g}=\frac{n_{1} n_{g 1} D+n_{2} n_{g 2}(1-D)}{\sqrt{D n_{1}{ }^{2}+(1-D) n_{2}{ }^{2}}}
$$

where $n_{1}$ and $n_{2}$ are the effective indices of the silicon and silica waveguides, respectively, $n_{g 1}$ and $n_{g 2}$ are the group index of the silicon and silica waveguides.

To investigate the performance of the integrated indexvariable OTTDL, we fabricated an array of forty SWG waveguides in SOI, see Fig. 1(a). The SWG waveguides are formed by alternating periodically segments of silicon and silica with a period of $\Lambda=250 \mathrm{~nm}$. Each waveguide in the array is $34 \mathrm{~mm}$ long and the duty cycles are varied in 1\% increments from $30 \%$ to $69 \%$. The waveguides are arranged in a serpentine configuration to reduce size; each bend includes two SWG tapers to transition between the SWG waveguide and solid core waveguide used as the waveguide bend, as shown in Fig. 1(b). The SWG waveguides are separated by $\sim 31.5 \mu \mathrm{m}$ to eliminate crosstalk. Generally, a separation of $10 \mu \mathrm{m}$ avoids coupling and crosstalk [29], which is small enough to ensure compactness. The array of waveguides occupies a total chip area of $\sim 6.5 \mathrm{~mm}$ $\times 8.7 \mathrm{~mm}$ or $56.55 \mathrm{~mm}^{2}$.

The chip is fabricated using electron beam lithography with a single etch at Applied Nanotools (ANT) Inc.. The SWG waveguides have a cross-section of $220 \mathrm{~nm} \times 500 \mathrm{~nm}$; they are covered by an index-matched cladding layer of thickness 2.2 $\mu \mathrm{m}$. Each SWG waveguide has an input and output taper for coupling to a nanowire waveguide of the same cross-section, as illustrated in Fig. 1(c). The SWG tapers are used for mode conversion between the SWG waveguide and the solid core waveguide [28]. The duty cycle of the taper is the same as the duty cycle of the SWG waveguide, and the thickness of waveguides is $220 \mathrm{~nm}$. The length of a taper is $50 \mu \mathrm{m}$.

We use an EDFA (KEOPSYS, CEFA-C-HG) as an amplified spontaneous emission (ASE) source and an optical spectrum analyzer (OSA, Ando AQ6317) to obtain the spectral response of each SWG waveguide, as shown in Fig. 1(d). We use a timeof-flight to measure the propagation time through the waveguides. The experimental setup for the time-of-flight measurement is illustrated in Fig. 1(e). A tunable laser (Keysight, 8164B) generates a continuous wave at $1550 \mathrm{~nm}$ with an output power of $\sim 6 \mathrm{dBm}$. The laser is modulated employing an electro-optic modulator (EOM, JDS Uniphase OC-192) driven by an RF signal of $10 \mathrm{GHz}$ (Anritsu, 69377B). After propagating through each SWG waveguide, the signals are amplified by an EDFA (Pritel, LNHPFA-30) and then detected and observed using a digital communications analyzer (DCA, Agilent Infiniium DCA-J 86100C). The incremental delays are extracted from the measured waveforms using the measured trace from the first waveguide as a reference.

Fig. 2(a) shows the spectra at the output of the waveguides as well as that of the input broadband source. The total fiber-tofiber loss depends on the coupling loss associated with the vertical grating couplers (VGCs) used for coupling in and out from the chip, the propagation loss in the SWG waveguide, and the losses associated with the waveguide bends (including the tapers and transition between SWG and solid core waveguides). Fig. 2(b) shows the fiber-to-fiber loss for the array of forty SWG waveguides; the average loss is $\sim 33 \mathrm{~dB}$, which includes a VGC coupling loss of $\sim 22 \mathrm{~dB}$ (the loss of a VGC-to-VGC pair is characterized separately using test structures). Fig. 2(c) shows the measured time delays in the waveguides, which increase linearly as a function of duty cycle (apart from a few waveguides which may have been impacted by fabrication errors). The average incremental time delay between consecutive SWG waveguides is $\sim 4.7 \mathrm{ps}$. The total time delay between the first and last SWG waveguides is $\sim 181.9 \mathrm{ps}$. 

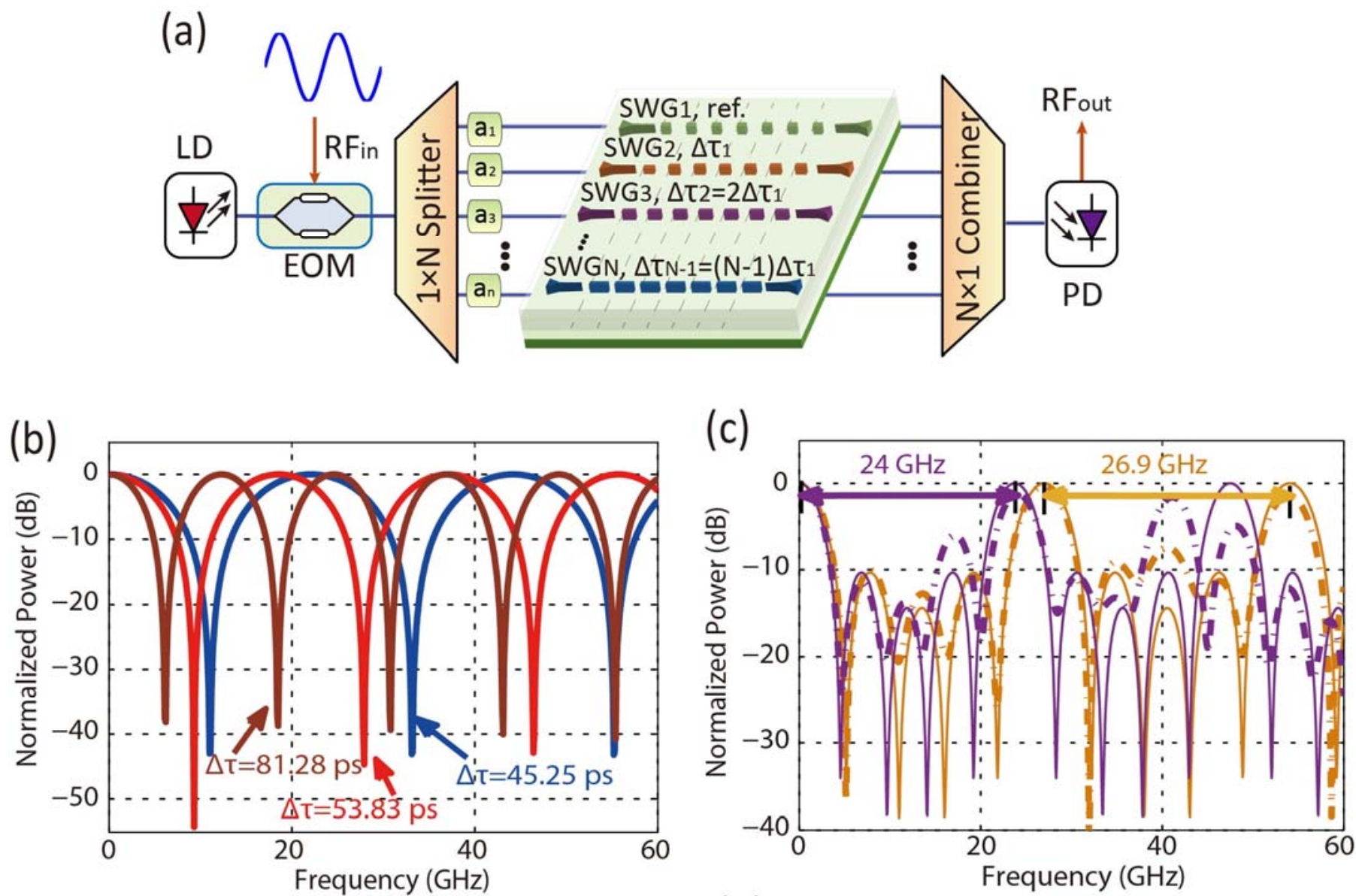

(d)

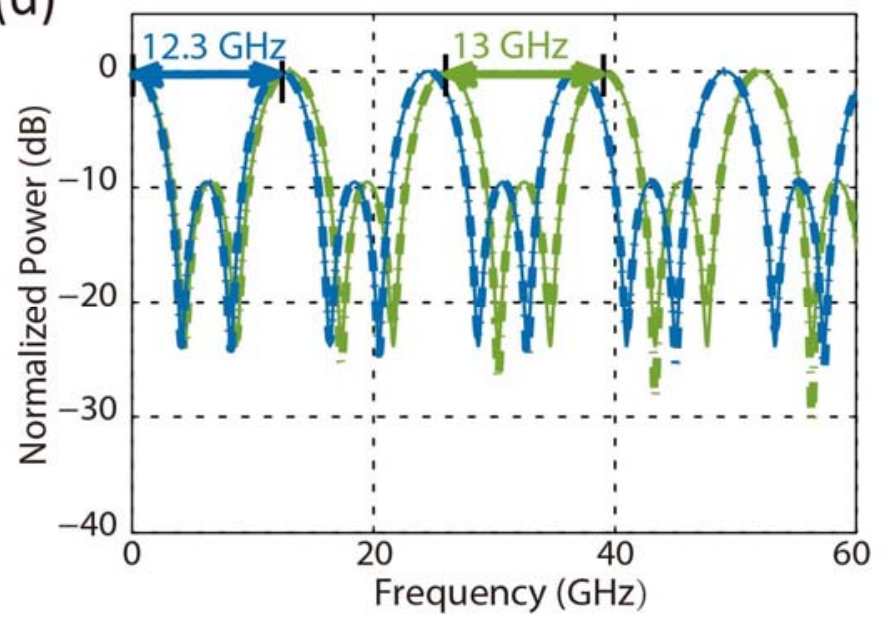

(e)

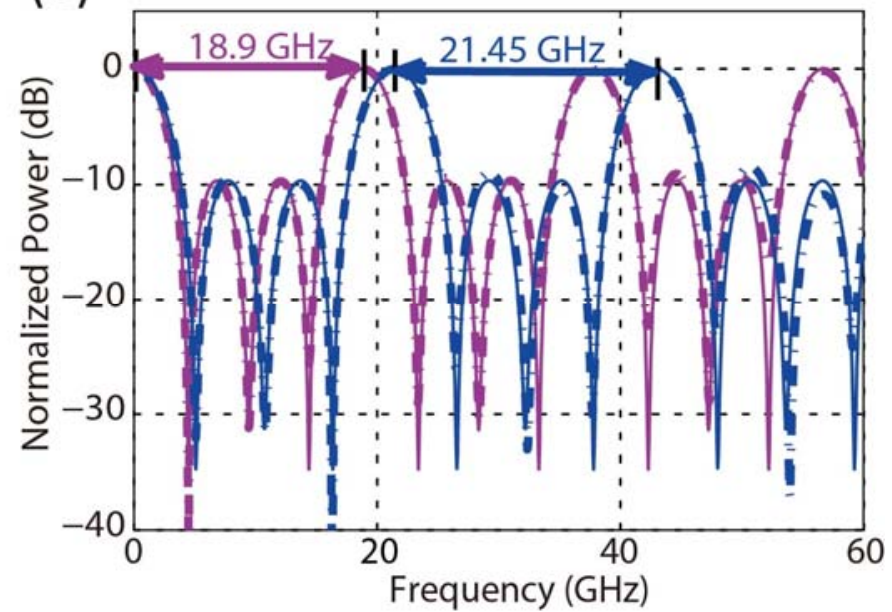

Fig. 3. Proposed $N$-tap MPF based on our SWG-based OTTDLs. (a) Schematic diagram. PD: photodetector. Simulated frequency responses of MPFs: (b) twotap, (c) three-tap, (d) four-tap, and (e) five-tap. By selecting different SWG waveguides, the frequency responses of the filters can be reconfigured by changing the FSR.

\section{APPLICATIONS FOR MWP}

We consider the performance of the fabricated OTTDLs and discuss a variety of applications for MWP. In particular, we focus on important applications such as MPFs, optical beamforming for phased array antennas, and multi-cavity OEOs.

\section{A. Application for microwave photonic filtering}

MPFs have been a topic of interest due to their wide range of applications in radar [30], communications [31], and real-time spectrum processing systems [32]. One popular implementation of an MPF is to use a delay line structure to obtain a finite impulse response [33]. Fig. 3(a) illustrates the generic layout of an $N$-tap transversal MPF using a single optical source and our array of forty SWG waveguides as a delay line MPF structure. The optical carrier from the laser source is modulated by a microwave signal using an electro-optic modulator (EOM). The modulated signal is then divided into $N$ paths by a $1 \times N$ splitter (to create $N$ taps); the taps experience different time 

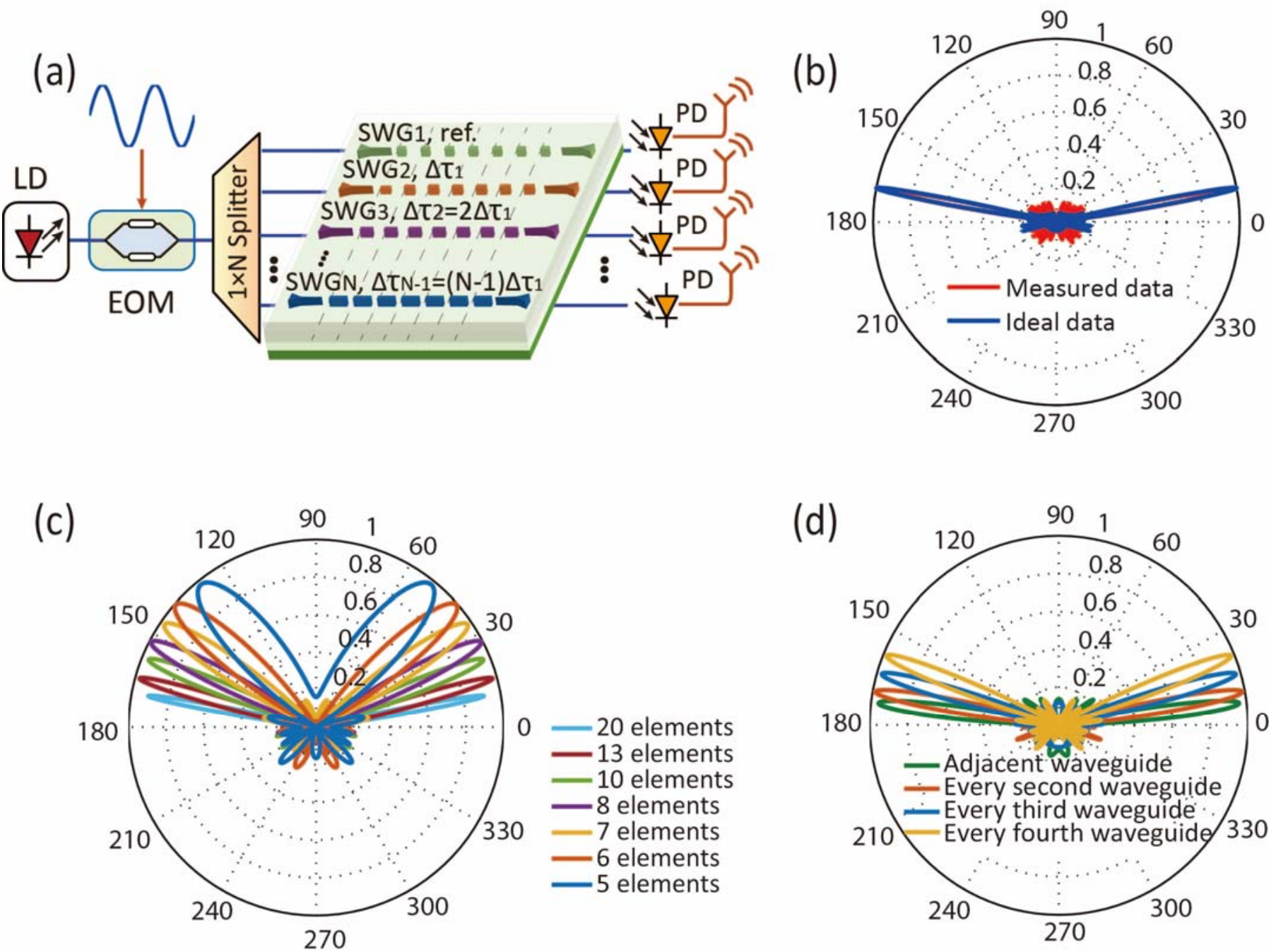

Fig. 4. Schematic of an optical beamforming phased antenna array based on the array of SWG waveguides. (a) Diagram for a uniform spaced N-element phased antenna array based on the fabricated OTTDLs. (b) Simulated antenna array factors for comparing the measured data and the ideal data. (c) Calculated antenna array factors for different numbers of antenna elements at $1550 \mathrm{~nm}$. (d) Simulated antenna array factors when a 10-element antenna array is fixed.

delays in the SWG waveguides before being recombined. Note the tap weights can be controlled using variable powers of taps, denoted by $a_{1}, a_{2}, \ldots, a_{n}$.

An $N$-tap MPF has a transfer function given by [34]:

$$
H(\omega)=\sum_{n=0}^{N} a_{n} e^{-j n \omega \Delta \tau},
$$

where $\omega$ is the angular frequency of the RF signal, $a_{n}$ is the coefficient of the $n^{\text {th }}$ tap, and $\Delta \tau$ is the time delay between consecutive taps. The delays from our SWG waveguides enable a simple implementation of a multi-tap MWP filter. Equation (2) shows that the MPF response is periodic. The FSR of the filter is inversely proportional to the time interval between adjacent taps $(\mathrm{FSR}=1 / \Delta \tau)$. Thus, tunability and reconfigurability of the MPF passband responses can be accomplished by control of $\Delta \tau$; reconfigurability of the passband shape can be implemented by varying the number of taps (waveguides used) and weight.

First, we simulate the frequency responses of uniform $N$-tap MPFs using the measured time delays from the SWG waveguides. The frequency responses of two-tap filters are shown in Fig. 3(b): when selecting two SWG waveguides, e.g., the $3^{\text {rd }}$ and $12^{\text {th }}$ waveguides, the $7^{\text {th }}$ and $18^{\text {th }}$ waveguides, or the $6^{\text {th }}$ and $23^{\text {rd }}$ waveguides, the corresponding differential time delays between adjacent samples (denoted as $\Delta \tau$ ) are $45.3 \mathrm{ps,}$ $53.8 \mathrm{ps}$, and $81.3 \mathrm{ps}$, respectively, which give rise to filter responses having a periodicity or free spectral range (FSR) of $22.1 \mathrm{GHz}, \sim 18.6 \mathrm{GHz}$, and $\sim 12.3 \mathrm{GHz}$.

Next, we calculate the frequency responses of three-tap, fourtap, and five-tap MPFs, as shown in Figs. 3(c), (d), and (e), respectively, by selecting three, four, and five SWG waveguides. For comparison, we also show the frequency responses of the ideal case (as dashed lines) where we use a uniform delay increment (obtained with the average value of measured differential delays $\Delta \tau$ ). As an example, for the threetap MPF, we begin by selecting the $6^{\text {th }}, 23^{\text {rd }}$, and $40^{\text {th }}$ SWG waveguides, the differential delays $\Delta \tau$ are $81.3 \mathrm{ps}$ and $81.5 \mathrm{ps}$, corresponding to an FSR of $13 \mathrm{GHz}$. On the other hand, by choosing the $7^{\text {th }}, 23^{\text {rd }}$ waveguide, and $39^{\text {th }}$ waveguides, the incremental delays are $77.2 \mathrm{ps}$ and $76.7 \mathrm{ps}$ resulting in an FSR of $12.3 \mathrm{GHz}$. Figs. 3(c), (d), and (e) illustrate additional examples of three-tap, four-tap, and five-tap MPFs: discrete reconfigurability can be realized by selecting different sets of 

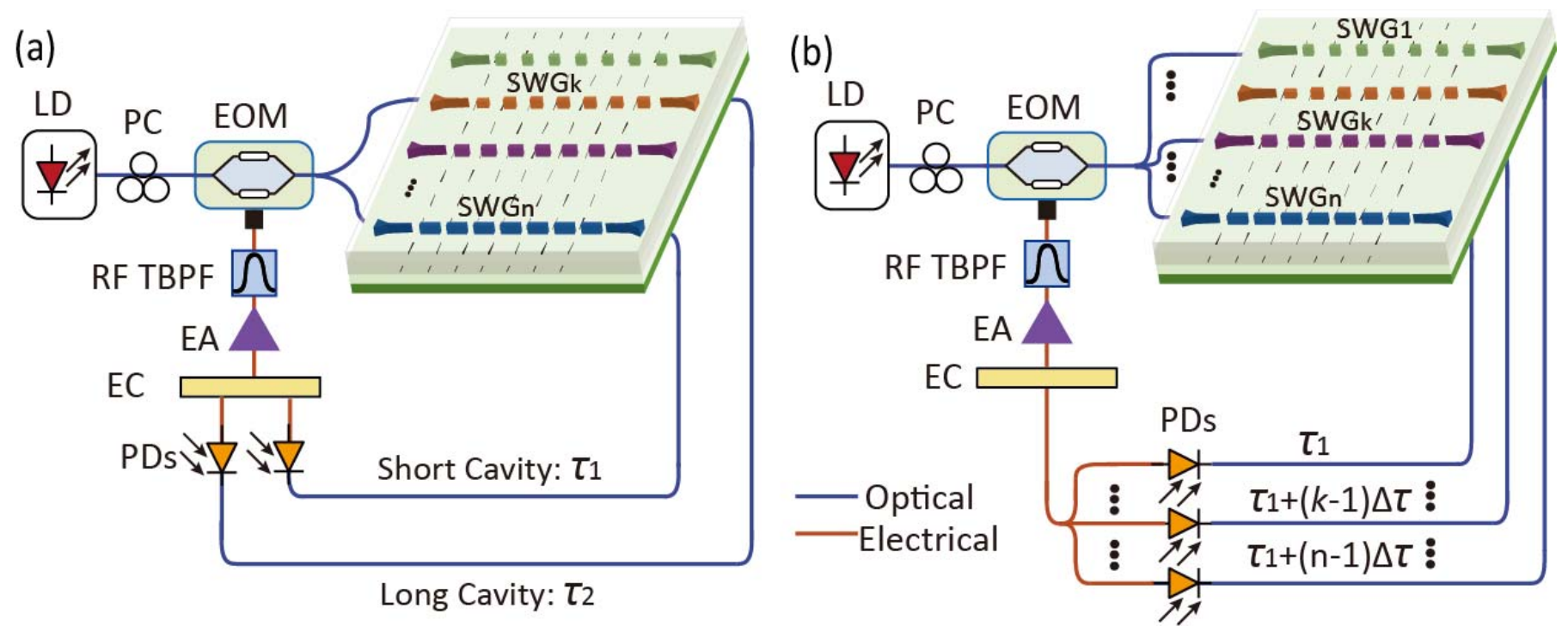

Fig. 5. Layout of the proposed OEOs based on the fabricated OTTDLs. (a) Unbalanced dual-cavity OEO using the fabricated OTTDL array. (b) Multi-cavity Vernier OEO using the fabricated OTTDL array. PC: polarization controller; EC: electrical coupler; EA: electrical amplifier; RF TBPF: RF tunable band-pass filter.

SWG waveguides since both the number of samples and the differential delay can be adjusted. There is a good agreement between the simulated responses based on the measured delays and ideal delays. Note that the reconfigurability of the filter can be realized as well by tuning the filter coefficients (i.e., $a_{1}$, $\left.a_{2}, \ldots, a_{n}\right)$ through controlling the power of each tap.

\section{B. Application for antenna beamforming}

Photonic technologies, such as OTTDLs, are of significant interest for optical beamforming in phased array antenna systems because they provide the possibility for remote control, allow ultra-wide operation bandwidth, greatly reduce the required footprint, and are immune to electromagnetic interference $[35,36]$. More importantly, OTTDLs avoid beam squint which can arise when using structures based on phase shifting elements.

The beam radiation direction is determined by the intensity and phase of radio waves emitted from antenna elements. In the case of a phased array antenna with its elements uniformly spaced and along a single axis, the normalized array factor of the phased array antenna with linearly spaced antenna elements can be expressed as $[36,37]$ :

$$
A F(\theta)=\frac{1}{N} \sum_{n=0}^{N-1} a_{n} e^{-j 2 \pi f n\left(\Delta \tau-\frac{d \sin \theta}{c}\right)},
$$

where $N$ is the number of antenna elements, $a_{n}$ is the complex coefficient of $n^{\text {th }}$ antenna element, $f$ is the RF, $\Delta \tau$ is the differential time delay, $d$ is the distance between two adjacent antenna elements, $\theta$ is the far-field angular coordinate, and $c$ is the light speed in vacuum. The array factor attains its maximum value at $\theta_{\max }$ when $\Delta \tau=d \sin \theta / c$. We can tune this beam-pointing angle $\theta_{\max }$ by changing the differential delay between adjacent samples.

Fig. 4(a) shows the schematic of an optical beamforming system that uses the array of SWG waveguides as an indexvariable OTTDL. The optical carrier emitted by a laser source is modulated by an RF signal through an EOM. The modulated signal is separated by a $1 \times N$ splitter before launching into the SWG waveguides to implement the delays. Each delayed signal is then directed towards a photodetector (PD) and the output drives an antenna element. The set of differential time delays associated with propagation in the different waveguides are translated into different beam-pointing angles of the phased array antenna.

We simulate the beamforming patterns (i.e., the array factors) for different delay increments assuming an optical carrier wavelength of $1550 \mathrm{~nm}$, an RF frequency of $10 \mathrm{GHz}$, and a separation between radiating elements of $1.5 \mathrm{~cm}$. First, we compare the patterns for 20 antenna elements where we use the measured and average incremental delays, see Fig. 4(b). The red line represents the array factor using the measured delays while the blue line shows the ideal case when we assume a uniform delay increment of $\sim 9.3 \mathrm{ps}$ (obtained with the average value of all measured delays for every second waveguide). In this case, the RF signal corresponding to the first delay line is taken as a reference, and the time delay differences $\Delta \tau_{1, n}$ are for the remaining delay lines with respect to the first delay line. Even though the measured incremental delays are not strictly uniform, we can see that the impact on the beamforming pattern is negligible though there is an increase in the sidelobes of the beam pattern. Fig. 4(c) depicts the array factors using different numbers of antenna elements and incremental delays. For example, we can consider using every second waveguide, which results in a 20 -element phased array antenna. We can also use fewer waveguides that are further separated in order to increase the incremental delay: choosing every third, fourth, fifth, sixth, seventh, and eighth waveguide results in $13,10,8,7,6$, and 5 elements, respectively. Clearly, the beamforming angle can be tuned discretely by simply choosing different delay lines: the angles are $10.4^{\circ}, 16.2^{\circ}, 21.8^{\circ}, 27.0^{\circ}, 33.8,41.1^{\circ}$, and $51.0^{\circ}$ for antennas with $20,13,10,8,7,6$, and 5 elements, respectively. It should be noted that since increasing the delay reduces the number of antenna elements, the directionality is reduced correspondingly. However, we can fix the number of 

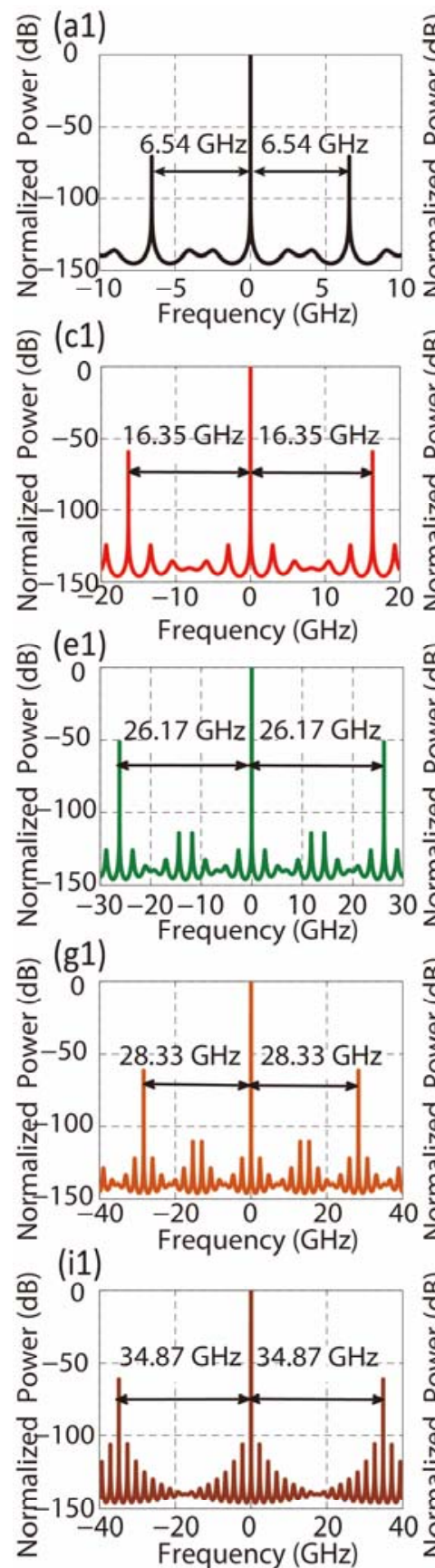
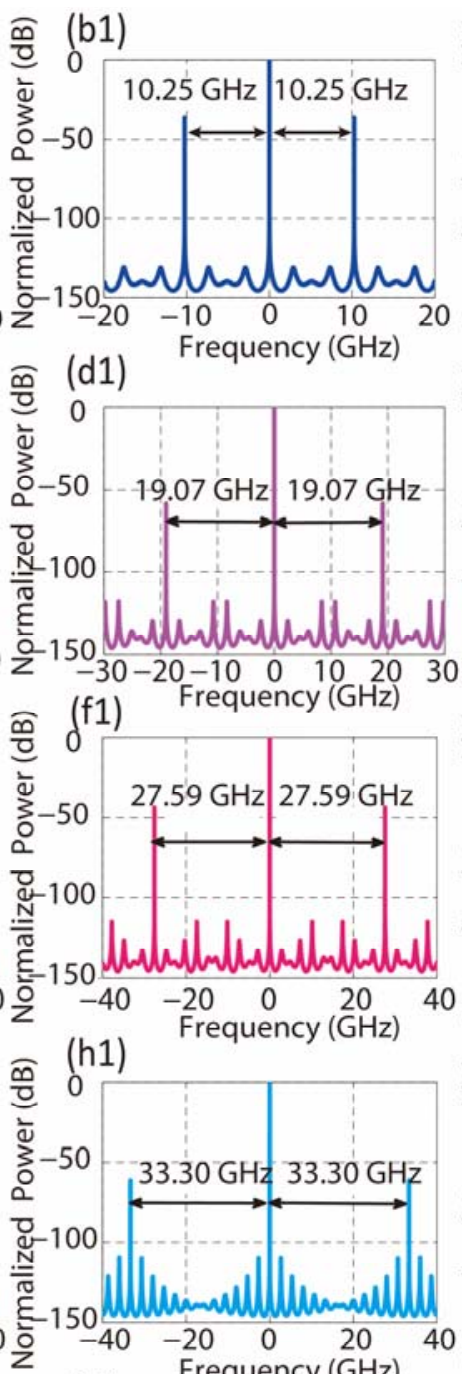

(j1)

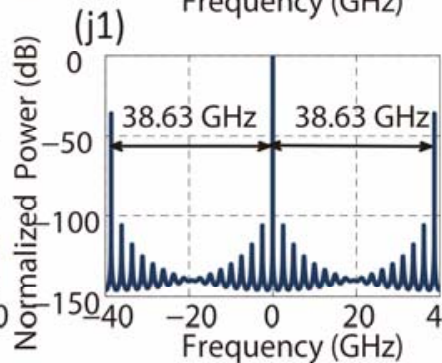

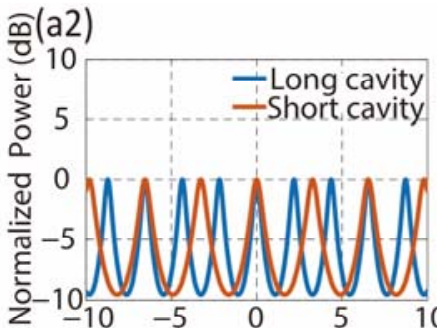
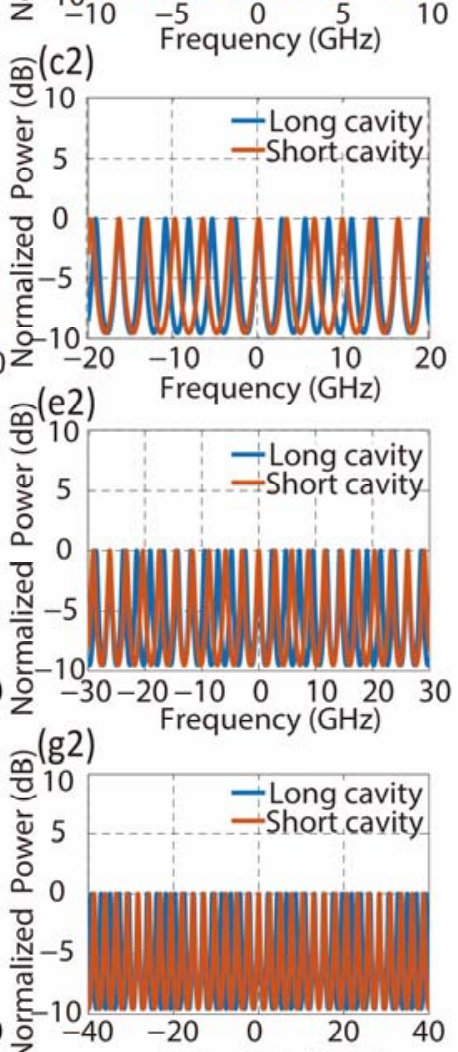

(i2)

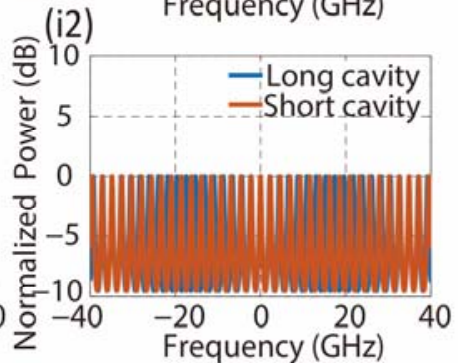

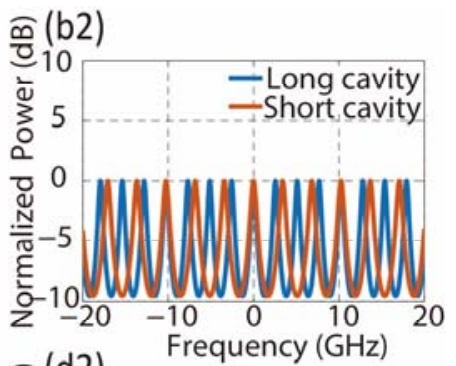
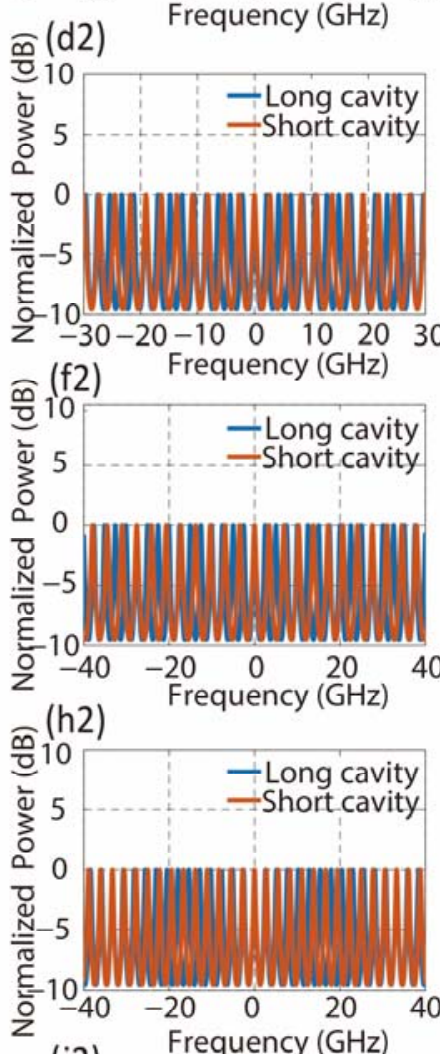

(j2) Frequency $(\mathrm{GHz})$

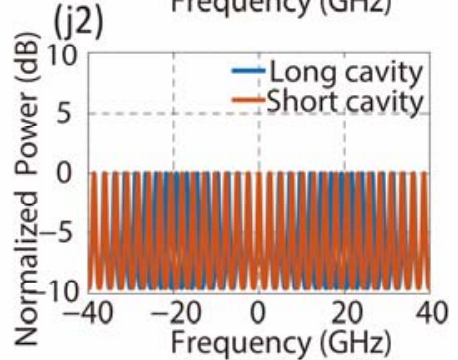

Fig. 6. (a1)-(j1) Simulated oscillation spectra of the unbalanced dual-cavity OEO with different oscillation frequencies: (a1) $6.54 \mathrm{GHz}$, (b1) $10.25 \mathrm{GHz}$, (c1) 16.35 GHz, (d1) $19.07 \mathrm{GHz}$, (e1) $26.17 \mathrm{GHz}$, (f1) $27.59 \mathrm{GHz}$, (g1) $28.33 \mathrm{GHz}$, (h1) $33.30 \mathrm{GHz}$, (i1) $34.87 \mathrm{GHz}$, and (j1) 38.63 GHz. (a2)-(j2) Simulated RF spectra associated with the individual cavities corresponding to the unbalanced dual-cavity OEOs in Figs. 6 (a1)-(j1).

waveguides used, e.g., 10, and then select the appropriate delays to tune the beamforming angle without impacting the directionality. Fig. 4(d) shows the simulated beamforming patterns for a 10-element antenna using every second, third, and fourth waveguide to obtain different time delays. The beamforming angle can be tuned discretely between $6.7^{\circ}, 10.3^{\circ}$, $16.1^{\circ}$, and $21.9^{\circ}$.

\section{Application for multi-cavity optoelectronic oscillators}

OEOs have attracted much attention due to their capacity to generate high-frequency and low-phase-noise microwave signals for applications in communications (e.g., radio over fiber systems), instrumentation (e.g., radar), and sensing [3840]. Usually, the oscillation of an OEO is produced through a feedback cavity that contains a modulator, an optical fiber delay, and a photodetector [41]. To obtain high spectral purity signals, the OEO needs a long fiber cavity, which generates many oscillation modes that are closely spaced in frequency. Therefore, selecting a single mode for oscillation requires a high-selectivity RF filter with narrow bandwidth. Multi-cavity OEOs have been investigated to remove the requirement of a narrowband RF filter [42].

Following a similar procedure as Yao and Maleki [43], the recursive relation that describes the circulating filed after the 

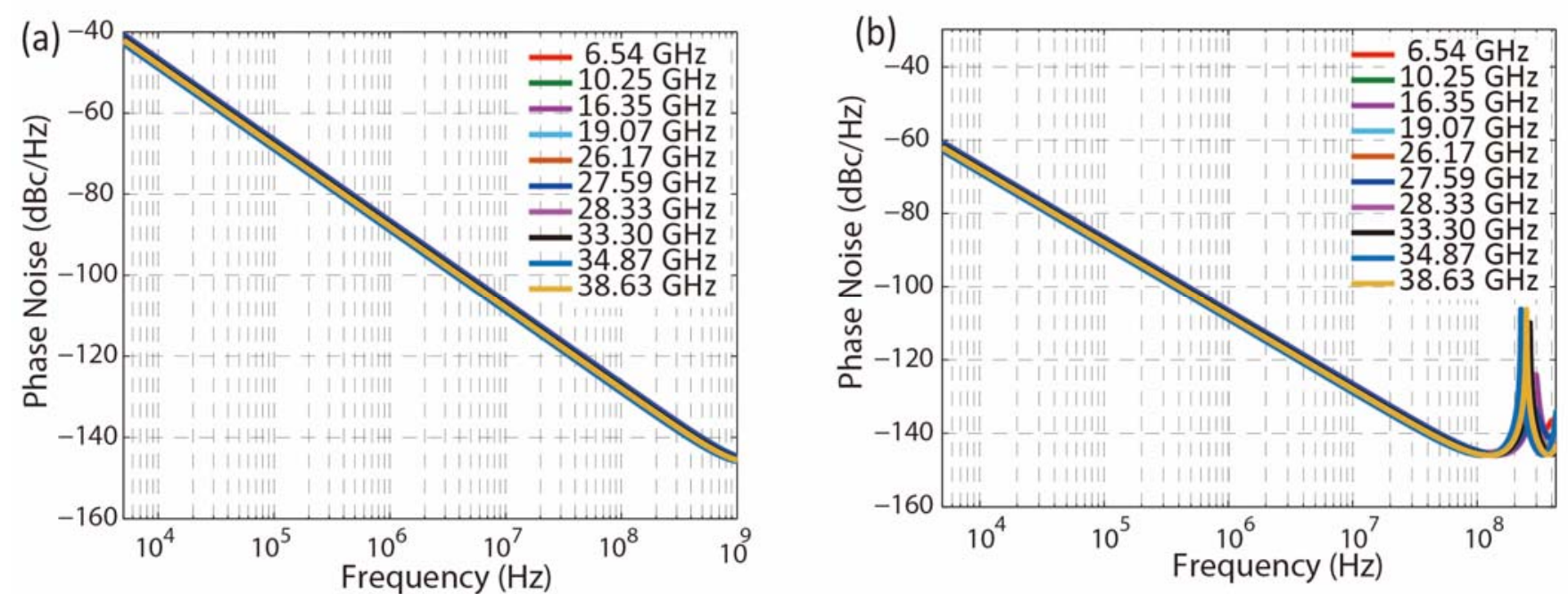

Fig. 7. Simulated phase noise spectra of the unbalanced dual-cavity OEOs at different oscillation frequencies. (a) Phase noise spectra for the cases shown in Figs. 6(a1)-(j1). (b) Phase noise spectra under the condition of increasing the lengths of the SWG waveguides by a factor of 10 (to obtain 10 times the delay).

$u^{\text {th }}$ round-trip can be given by:

$$
\tilde{V}_{u}(\omega)=\left[\sum_{k=1}^{N}\left|g_{k}\right| e^{j\left(\omega \tau_{k}+\phi_{k}\right)}\right] \tilde{V}_{u-1}(\omega),
$$

where $\tilde{V}_{u}(\omega)$ is the complex amplitude of the circulating filed after $u^{t h}$ round trip and $g_{k}$ is the complex gain of $k^{\text {th }}$ cavity. If the starting voltage of oscillation is $G_{a} \tilde{V}_{i n}(\omega)$, where $G_{a}$ is the gain of the RF amplifier, the total of all circulating fields is:

$$
\tilde{V}_{\text {out }}(\omega)=\sum_{u=0}^{\infty} \tilde{V}_{u}(\omega)=\frac{G_{a} \tilde{V}_{i n}(\omega)}{1-\sum_{k=1}^{N}\left|g_{k}\right| e^{j\left(\omega \tau_{k}+\phi_{k}\right)}} .
$$

The corresponding RF power is:

$$
P(\omega)=\frac{\left|\tilde{V}_{\text {out }}(\omega)\right|^{2}}{2 R}=\frac{G_{a}{ }^{2}\left|\tilde{V}_{\text {in }}(\omega)\right|^{2} / 2 R}{\left|1-\sum_{k=1}^{N}\right| g_{k}\left|e^{j\left(\omega \tau_{k}+\phi_{k}\right)}\right|^{2}},
$$

where $R$ is the load impedance and:

$$
\Phi_{k}(\omega)=\omega \tau_{k}+\phi_{k},
$$

where $\tau_{k}$ is the time delay and $\phi_{k}$ is the phase factor of complex gain $g_{k}$.

For the oscillations to start collectively in all cavities and start from noise [43], we consider a simple case that the gain coefficients have equal value in all cavities $\left|g_{k}\right|=g=1 / N$, $N$ is the number of cavities in the multi-cavity OEO.

The phase noise model of the multi-cavity OEO is also investigated. The power spectral density of the mode oscillating at $\omega_{0}$ can be expressed as [43]:

$$
S_{R F}\left(f^{\prime}\right)=\frac{G_{a}{ }^{2} \rho_{N}{ }^{2} / P_{o s C}}{\left|1-\sum_{k=1}^{N}\right| g_{k}\left|e^{\mathrm{j}\left(2 \pi f^{\prime} \tau_{k}\right)}\right|^{2}},
$$

where $\omega_{0}$ is the oscillation angular frequency, $f^{\prime}=\frac{\omega-\omega_{0}}{2 \pi}$ represents the offset frequency from the RF carrier, $P_{o s c}$ is the RF oscillation power, $\rho_{N}$ represents all the technical noise sources of the OEO [44], including thermal noise, the laser's relatively intensity noise and, where applicable, amplified spontaneous emission noise. $\rho_{N}$ is usually reported by a value in the range of -180 to $-160 \mathrm{dBc} / \mathrm{Hz}$.

For starting oscillations of a dual-cavity OEO, the oscillation frequency must verify [43]:

$$
f_{0}=\frac{k}{\tau_{1}}=\frac{n}{\tau_{2}},
$$

where $k$ and $n$ are integers, and $\tau_{1}$ and $\tau_{2}$ are the cavity time delays of two cavities, respectively. The mode spacing $\Delta f$ is determined by the shorter cavity: $\Delta f=\frac{1}{\tau_{1}}$. In addition, the resonance linewidth is imposed by the longer cavity.

Equation (9) is employed to determine the required $\tau_{1}$ and $\tau_{2}$, then we select two SWG waveguides that provide these delays. For example, we choose the $5^{\text {th }}$ and $38^{\text {th }}$ waveguides whose delays are $305.8 \mathrm{ps}$ and $458.9 \mathrm{ps}$, respectively, leading to $k=2, n=3$ and an oscillation frequency $f_{0} \sim 6.54 \mathrm{GHz}$. By the same token, Fig. 6(a1)-(j1) reports the oscillation frequency $f_{0}$ when different pairs of SWG waveguides are chosen; this shows how the output frequency of an unbalanced dual-cavity OEO can be tuned discretely.

A multi-cavity OEO can be implemented by using $N$ SWG waveguides as the corresponding $N$ cavities of the OEO. The incremental delay between adjacent cavities is given by $\Delta \tau_{k}$. In this case, (9) can be expressed as [43]:

$$
\Phi_{k}(\omega)=\omega \tau_{1}+(k-1) \omega \Delta \tau_{k}+\phi_{k}(k=1,2, \ldots, N) .
$$

A simple case is when $\phi_{k}=0 \forall k$ and to start oscillation, the oscillation frequency must satisfy:

$$
f_{0}=\frac{m}{\tau_{1}}(m=1,2, \ldots) .
$$

Note that the oscillation frequency is set by the value of $\tau_{1}$. From the Vernier effect and to have constructive interference at $f_{0}$, the incremental delays $\Delta \tau_{k}$ must satisfy [43]:

$$
f_{0}=\frac{1}{\Delta \tau_{k}} \text {. }
$$

Note that oscillations start only when (11) and (12) are satisfied simultaneously.

Fig. 5(a) illustrates the schematic of an unbalanced dualcavity OEO that uses our array of SWG waveguides as an index-variable OTTDL. The optical carrier from a laser source is introduced into an EOM and the output is split and sent through two selected SWG waveguides to impose different 
(a)

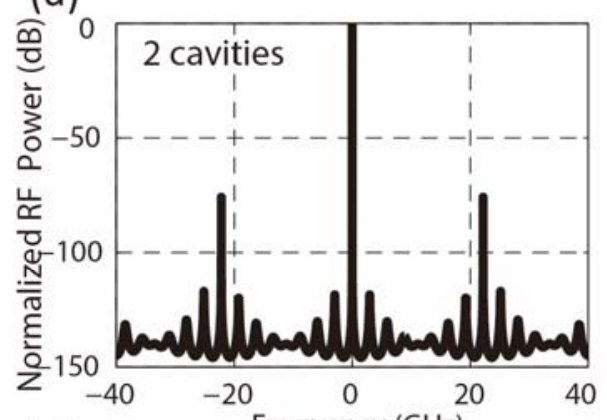

(d)
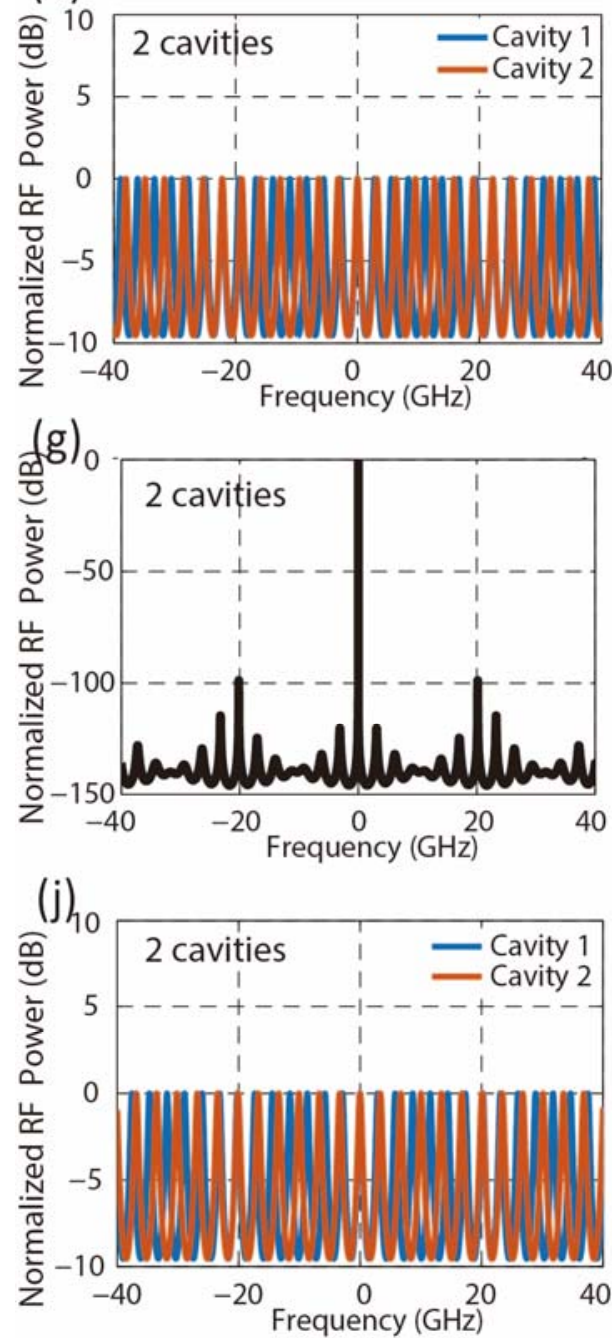

(b)

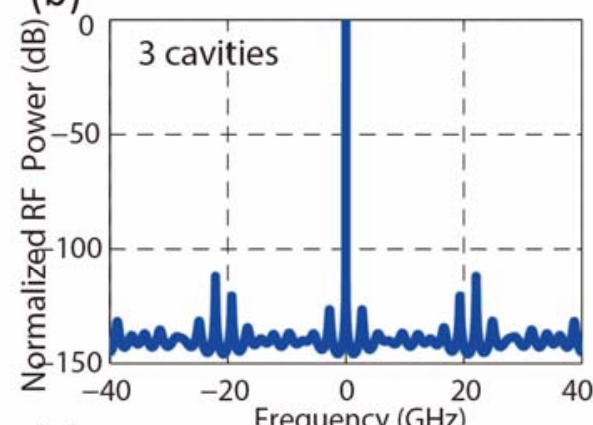

(e)
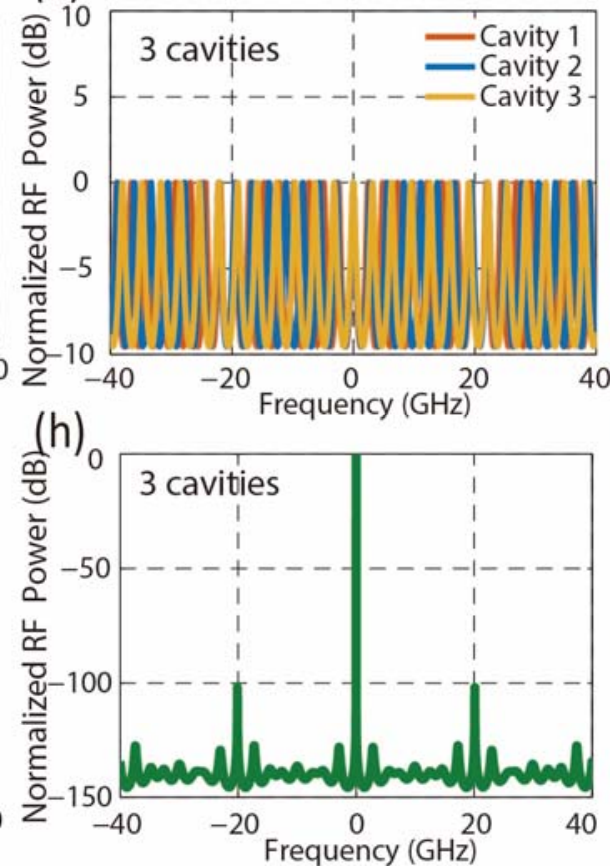

(k)

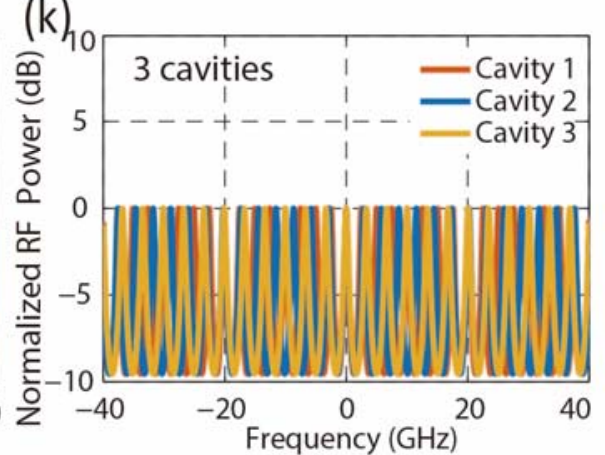

(c)

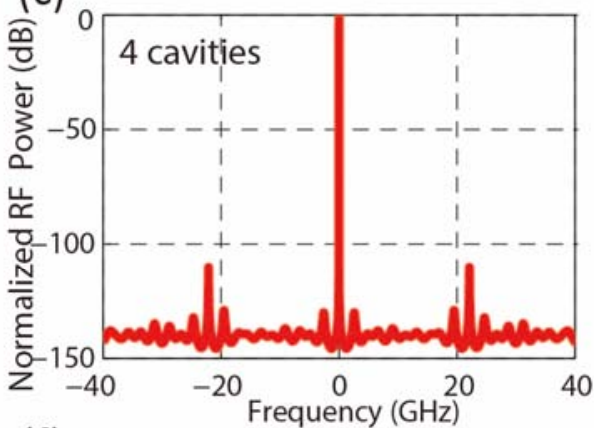

(f)

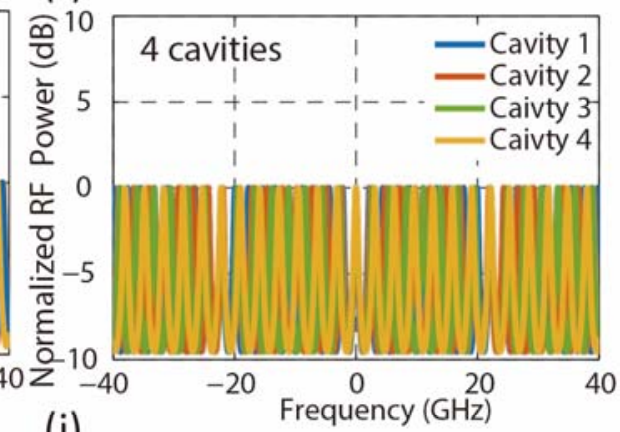

(i)
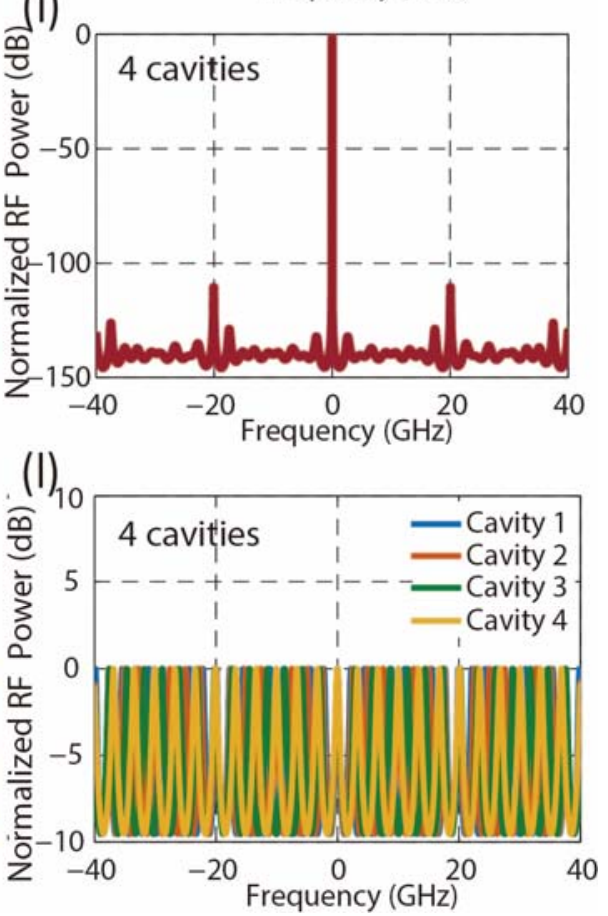

Fig. 8. Simulated oscillation spectra for the multi-cavity Vernier OEOs. Oscillation frequency of $\sim 22.2 \mathrm{GHz}$ for (a) 2 cavities, (b) 3 cavities, and (c) 4 cavities. (d)-(f) Corresponding spectra of each cavity comprising the multi-cavity Vernier OEO. (g)-(i) Oscillation frequency of $\sim 20 \mathrm{GHz}$ for (g) 2 cavities, (h) 3 cavities, and (i) 4 cavities. (j)-(1) Corresponding spectra of each cavity comprising the multi-cavity Vernier OEO.

propagation times before being detected. The electrical output of the PD is then fed back to the electric port of the EOM after amplification.

Figs. 6(a1)-(j1) show the calculated oscillation spectra for an unbalanced dual-cavity OEO using the measured delays for different pairs of SWG waveguides. For comparison, the individual spectra from each cavity are also plotted as shown in Figs. 6(a2)-(j2). Note that since the measured delays for the chosen waveguides do not satisfy (9) exactly, the amplitudes of the generated tones are attenuated. Due to the small incremental delays provided by our SWG waveguide-based OTTDL, the FSR of the oscillation spectra for the unbalanced dual-cavity OEOs is typically on the order of GHz. As a result, a highly selective RF filter is not necessary for mode selection, in contrast with other approaches [43]. By choosing different waveguide pairs to obtain different delays, a discretely tunable microwave signal from $6.54 \mathrm{GHz}$ to $38.63 \mathrm{GHz}$ can be obtained readily. Moreover, even if the delays result in the two cavities 

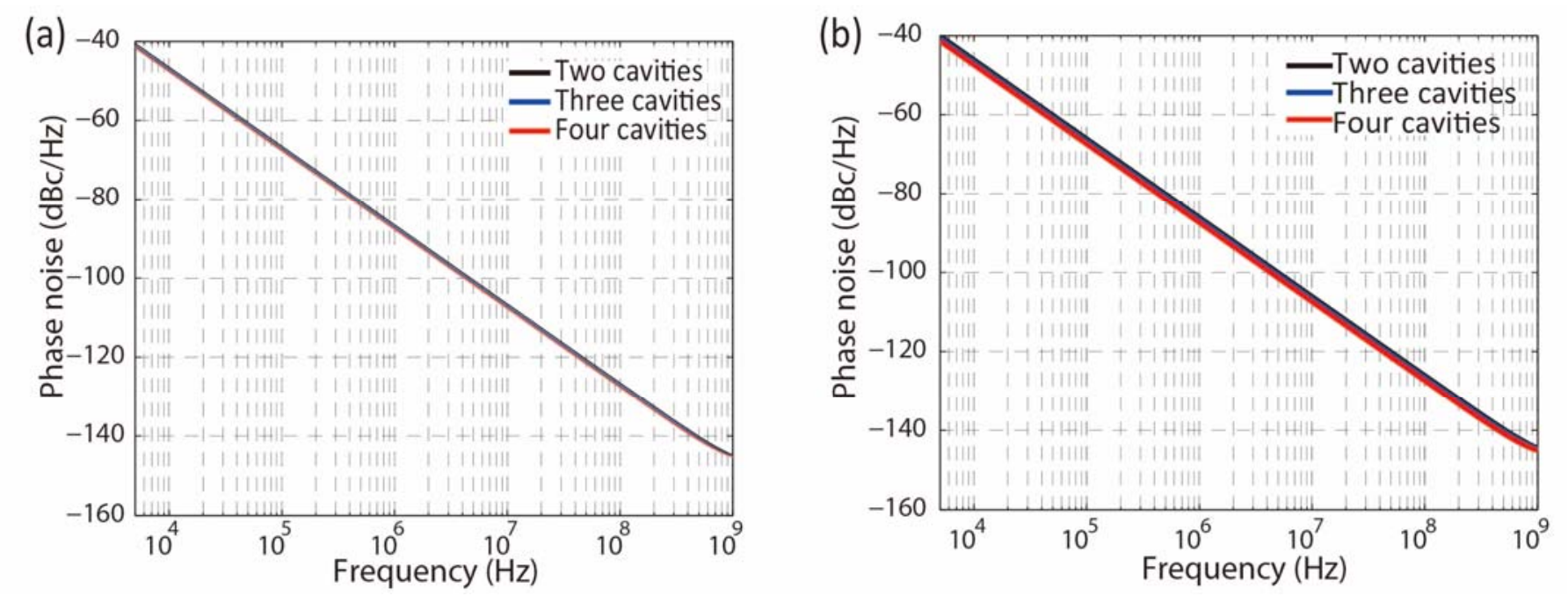

Fig. 9. Simulated phase noise spectra of the multi-cavity OEOs. (a) Oscillation frequency of $\sim 22.2 \mathrm{GHz}$. (b) Oscillation frequency of $\sim 20 \mathrm{GHz}$

having slightly different central frequencies, the impact is negligible and the sidemode suppression is still high.

We also investigate the phase noise spectra of the unbalanced dual-cavity OEOs, assuming a standard noise $\rho_{N}=-180$ $\mathrm{dBc} / \mathrm{Hz}$, an $\mathrm{RF}$ amplifier gain of $G_{a}=10 \mathrm{dBm}$, and an oscillation power of $P_{o s c}=10 \mathrm{dBm}$. Fig. 7(a) shows that the phase noise in all cases is similar because using larger delays has a negligible effect on the resonance quality factor, and the phase noise is largely independent of oscillation frequency. However, since our delays are in the ps range, we suffer more in terms of phase noise; using longer SWG waveguides to increase the delay will improve the phase noise. Note that for the unbalanced dual-cavity OEO, the phase noise is determined by that of the longer cavity [42]. By extending the basic cavity length, for example by increasing the length of the SWG waveguides in the OTTDL by a factor of ten, the phase noise characteristics can be improved by $20 \mathrm{~dB}$, as shown in Fig. 7(b).

Fig. 5(b) depicts the schematic of a multi-cavity OEO where the different SWG waveguides are employed to implement cavities with different delays. For example, from our measured data, we can choose SWG waveguides with single-cavity delays of $315.8 \mathrm{ps}, 360.4 \mathrm{ps}, 410.3 \mathrm{ps}$, and $451.6 \mathrm{ps}$. Figs. 8(a), (b), and (c) show the oscillation spectra for the oscillation frequency of $\sim 22.2 \mathrm{GHz}$ when we use these four SWG waveguides and vary the number of cavities ranges from two to four. The oscillation spectra of each cavity comprising the multi-cavity OEO are also plotted in Figs. 8(d), (e), and (f).

To obtain a tunable multi-cavity OEO, we change the delays by selecting different groups of SWG waveguides. For example, we can choose waveguides with single-cavity delays of 298.6 ps, 346.1 ps, 398.8 ps, and 451.6 ps. Figs. 8(g), (h), and (i) show the oscillation spectra for an oscillation frequency of $\sim 20 \mathrm{GHz}$ and the number of cavities ranges from two to four. As with the unbalanced dual-cavity OEO, the FSRs of the multi-cavity OEOs are in the $\mathrm{GHz}$ range thus, again, a highly selective filter is not required for mode selection. Moreover, by increasing the number of cavities, the spurious modes of the multi-cavity OEO can be suppressed. But as with the unbalanced dual-cavity OEO, since the measured delays do not satisfy (11) and (12) exactly, the amplitudes of the generated tones are attenuated. In Fig. 9, we plot the phase noise of the multi-cavity OEOs for operating frequencies of $\sim 22.2 \mathrm{GHz}$ and $\sim 20 \mathrm{GHz}$ (assuming, as before, a standard noise $\rho_{N}=-180 \mathrm{dBc} / \mathrm{Hz}$, an RF amplifier gain of $G_{a}=10 \mathrm{dBm}$, and an oscillation power of $P_{\text {osc }}=10 \mathrm{dBm}$. The results show that the phase noise for the two oscillating frequencies are similar.

\section{SUMmary AND Discussion}

We have proposed and designed experimentally an OTTDL based on an array of forty SWG waveguides in SOI, where each waveguide is $34 \mathrm{~mm}$ long. By controlling the duty cycles which are varied in $1 \%$ increments from $30 \%$ to $69 \%$, an average incremental delay of $\sim 4.7 \mathrm{ps}$ can be obtained. Our proposed method provides a promising solution for achieving large-scale integration of OTTDL devices on photonic chips. We demonstrate the use of the OTTDLs to implement $N$-tap MPFs with discrete frequency tunability and reconfigurability. Compared with the multicore fiber-based MPF [26, 27], the MPF using the SWG waveguide-based OTTDL can take advantage of on-chip transfer printed tunable gain semiconductor optical amplifiers (SOAs) [45] to control the tap amplitude values and provides a significantly more compact implementation. These transfer printed SOAs can allow us to overcome splitting losses when using many parallel SWG waveguides and propagation losses if the length of the SWG waveguides are increased to provide a greater differential delay. We also show the use of the OTTDL for antenna beamforming: by simply choosing different incremental delays (corresponding to different SWG waveguide), we can discretely tune the beamforming angle without incurring any beam-squint effects. Finally, we use the SWG waveguide-based OTTDL to implement different multi-cavity OEO possibilities without requiring high-selectivity RF filters.

While we only demonstrated discrete tuning of the MPF responses, beamforming patterns, and OEO oscillation frequencies by exploiting the space diversity provided by the different SWG waveguides, continuous tuning should be possible by changing the wavelength of the optical carrier as observed using heterogeneous multicore fibers [26, 27]. In 
particular, by operating closer to the SWG waveguide band edge, we may be able to take advantage of the increased dispersion in order to have different incremental values of dispersion and hence group delays as the wavelength of the optical carrier is tuned.

Increasing the number of SWG waveguides can provide more options for the number of taps for MPFs and phased antenna array elements, as well as a greater number of oscillation frequencies in dual-cavity and multi-cavity OEOs. It should be possible to increase the number of the SWG waveguides by reducing the increment in duty cycle. For example, using 80 SWG waveguides with duty cycles ranging from $30 \%$ to $69 \%$ can be obtained using an increment of $0.5 \%$ (which corresponds to a difference in the SWG segments of $\sim 1.25 \mathrm{~nm}$ and is achievable within the limits of ebeam lithography). Increasing the range of duty cycles will have limits, though. In particular, it has been shown that a larger duty cycle (i.e., $70 \%$ ) involves significantly higher taper and propagation losses $[29,46]$.

Based on our findings, we believe that our SWG waveguidebased OTTDL offers a versatile and compact solution to enable a wide range of integrated MWP signal processing functions for enhanced radar, communications, sensing, and instrumentation applications. Beyond MWP, this approach can be extended to perform additional optical signal processing applications that require different values of the group delay.

\section{REFERENCES}

[1] J. Capmany, J. Mora, I. Gasulla, J. Sancho, J. Lloret, and S. Sales, "Microwave photonic signal processing," J. Light. Technol., vol. 31, no. 4, pp. 571-586, 2012.

[2] A. J. Seeds, "Microwave photonics," IEEE Trans. Microw. Theory Tech., vol. 50, no. 3, pp. 877-887, 2002.

[3] S. Iezekiel, M. Burla, J. Klamkin, D. Marpaung, and J. Capmany, "RF engineering meets optoelectronics: Progress in integrated microwave photonics," IEEE Microw. Mag., vol. 16, no. 8, pp. 28-45, 2015.

[4] A. J. Seeds, and K. J. Williams, "Microwave photonics," J. Light. Technol., vol. 24, no. 12, pp. 4628-4641, 2006.

[5] J. Capmany, and D. Novak, "Microwave photonics combines two worlds," Nat. Photonics, vol. 1, no. 6, pp. 319-330, 2007.

[6] A. Pasquazi, M. Peccianti, B. E. Little, S. T. Chu, D. J. Moss, and R. Morandotti, "Stable, dual mode, high repetition rate mode-locked laser based on a microring resonator," Opt. Express, vol. 20, no. 24, pp. 27355-27363, 2012.

[7] D. Marpaung, C. Roeloffzen, R. Heideman, A. Leinse, S. Sales, and J. Capmany, "Integrated microwave photonics," Laser Photonics Rev., vol. 7, no. 4, pp. 506-538, 2012.

[8] D. Marpaung, M. Pagani, B. Morrison, and B. J. Eggleton, "Nonlinear integrated microwave photonics," J. Light. Technol., vol. 32, no. 20, pp. 3421-3427, 2014.

[9] C. G. H. Roeloffzen, L. Zhuang, C. Taddei, A. Leinse, R. G. Heideman, P. W. L. Van Dijk, R. M. Oldenbeuving, D. A. I. Marpaung, M. Burla, and K. J. Boller, "Silicon nitride microwave photonic circuits," Opt. Express, vol. 21, no. 19, pp. 22937-22961, 2013.

[10] L. R. Chen, "Silicon photonics for microwave photonics applications," J. Light. Technol., vol. 35, no. 4, pp. 824-835, 2016.

[11] R. A. Minasian, E. H. W. Chan, and X. Yi, "Microwave photonic signal processing," Opt. Express, vol. 21, no. 19, pp. 22918-22936, 2013.

[12] I. Frigyes, and A. J. Seeds, "Optically generated true-time delay in phased-array antennas," IEEE Trans. Microw. Theory Tech., vol. 43, no. 9, pp. 2378-2386, 1995.

[13] J.-D. Shin, B.-S. Lee, and B.-G. Kim, “Optical true time-delay feeder for $\mathrm{X}$-band phased array antennas composed of $2 /$ spl times/2 optical MEMS switches and fiber delay lines," IEEE Photon. Technol. Lett., vol. 16, no. 5, pp. 1364-1366, 2004.
[14] X. Ye, F. Zhang, and S. Pan, "Optical true time delay unit for multibeamforming," Opt. Express, vol. 23, no. 8, pp. 10002-10008, 2015.

[15] J. L. Cruz, and B. Ortega, "Chirped fibre Bragg gratings for phased-array antennas," Electron. Lett., vol. 33, no. 7, pp. P.545-546, 1997.

[16] Y. Liu, J. Yao, and J. Yang, "Wideband true-time-delay unit for phased array beamforming using discrete-chirped fiber grating prism," Opt. Commun., vol. 207, no. 1-6, pp. 177-187, 2002.

[17] K. Y. Song, M. Gonzalez-Herraez, and L. Thévenaz, "Observation of pulse delaying and advancement in optical fibers using stimulated Brillouin scattering," Opt. Express, vol. 13, no. 1, pp. 82-88, 2005.

[18] S. Chin, L. Thévenaz, J. Sancho, S. Sales, J. Capmany, P. Berger, J. Bourderionnet, and D. Dolfi, "Broadband true time delay for microwave signal processing, using slow light based on stimulated Brillouin scattering in optical fibers," Opt. Express, vol. 18, no. 21, pp. 2259922613, 2010.

[19] H. Lee, T. Chen, J. Li, O. Painter, and K. J. Vahala, "Ultra-low-loss optical delay line on a silicon chip," Nat. Commun., vol. 3, pp. 1-7, 2012.

[20] R. L. Moreira, J. Garcia, W. Li, J. Bauters, J. S. Barton, M. J. Heck, J. E. Bowers, and D. J. Blumenthal, "Integrated ultra-low-loss 4-bit tunable delay for broadband phased array antenna applications," IEEE Photon. Technol. Lett., vol. 25, no. 12, pp. 1165-1168, 2013.

[21] J. Xie, L. Zhou, Z. Zou, J. Wang, and J. Chen, "Continuously tunable reflective-type optical delay lines using microring resonators," Opt. Express, vol. 22, no. 1, pp. 817-823, 2014.

[22] R. Halir, P. J. Bock, P. Cheben, A. Ortega-Moñux, C. Alonso-Ramos, J. H. Schmid, J. Lapointe, D. X. Xu, J. G. Wangüemert-Pérez, and Í. I. Molina-Fernández, "Waveguide sub-wavelength structures: a review of principles and applications," Laser Photonics Rev., vol. 9, no. 1, pp. 2549, 2015.

[23] P. Cheben, R. Halir, J. H. Schmid, H. A. Atwater, and D. R. Smith, "Subwavelength integrated photonics," Nature, vol. 560, no. 7720, pp. 565-572, 2018.

[24] P. J. Bock, P. Cheben, J. H. Schmid, J. Lapointe, A. Delâge, S. Janz, G. C. Aers, D.-X. Xu, A. Densmore, and T. J. Hall, "Subwavelength grating periodic structures in silicon-on-insulator: a new type of microphotonic waveguide," Opt. Express, vol. 18, no. 19, pp. 20251-20262, 2010.

[25] I. Gasulla, and J. Capmany, "Microwave photonics applications of multicore fibers," IEEE Photon. J., vol. 4, no. 3, pp. 877-888, 2012.

[26] S. García, and I. Gasulla, "Dispersion-engineered multicore fibers for distributed radiofrequency signal processing," Opt. Express, vol. 24, no. 18, pp. 20641-20654, 2016.

[27] S. García, M. Ureña, and I. Gasulla, "Heterogeneous multicore fiber for optical beamforming." in 2019 International Topical Meeting on Microwave Photonics (MWP), Ottawa, ON, CAN, 2019, pp. 1-4.

[28] J. Wang, R. Ashrafi, R. Adams, I. Glesk, I. Gasulla, J. Capmany, and L. R. Chen, "Subwavelength grating enabled on-chip ultra-compact optical true time delay line," Sci. Rep., vol. 6, pp. 30235, 2016.

[29] V. Donzella, A. Sherwali, J. Flueckiger, S. T. Fard, S. M. Grist, and L. Chrostowski, "Sub-wavelength grating components for integrated optics applications on SOI chips," Opt. Express, vol. 22, no. 17, pp. 2103721050,2014

[30] X. Han, E. Xu, W. Liu, and J. Yao, "Tunable dual-passband microwave photonic filter using orthogonal polarization modulation," IEEE Photon. Technol. Lett., vol. 27, no. 20, pp. 2209-2212, 2015.

[31] J. Capmany, B. Ortega, and D. Pastor, "A tutorial on microwave photonic filters,” J. Light. Technol., vol. 24, no. 1, pp. 201-229, 2006.

[32] M. A. Laso, T. Lopetegi, M. J. Erro, D. Benito, M. J. Garde, M. A. Muriel, M. Sorolla, and M. Guglielmi, "Real-time spectrum analysis in microstrip technology," IEEE Trans. Microw. Theory Tech., vol. 51, no. 3, pp. 705-717, 2003.

[33] Y. Zhang, and S. Pan, "Tunable multitap microwave photonic filter with all complex coefficients," Opt. Lett., vol. 38, no. 5, pp. 802-804, 2013.

[34] J. Capmany, B. Ortega, D. Pastor, and S. Sales, "Discrete-time optical processing of microwave signals," J. Light. Technol., vol. 23, no. 2, pp. 702-723, 2005.

[35] M. Pelusi, F. Luan, T. D. Vo, M. R. Lamont, S. J. Madden, D. A. Bulla, D.-Y. Choi, B. Luther-Davies, and B. J. Eggleton, "Photonic-chip-based radio-frequency spectrum analyser with terahertz bandwidth," Nat. Photonics, vol. 3, no. 3, pp. 139-143, 2009.

[36] Phased array antenna handbook: Artech house, Norwood, Co., USA, 2017.

[37] S. Blais, and J. Yao, "Photonic true-time delay beamforming based on superstructured fiber Bragg gratings with linearly increasing equivalent chirps," J. Light. Technol., vol. 27, no. 9, pp. 1147-1154, 2009. 
[38] D. Strekalov, D. Aveline, N. Yu, R. Thompson, A. B. Matsko, and L. Maleki, "Stabilizing an optoelectronic microwave oscillator with photonic filters," J. Light. Technol., vol. 21, no. 12, pp. 3052, 2003.

[39] X. Zou, X. Liu, W. Li, P. Li, W. Pan, L. Yan, and L. Shao, "Optoelectronic oscillators (OEOs) to sensing, measurement, and detection," IEEE J. Quantum Electron., vol. 52, no. 1, pp. 1-16, 2015.

[40] J. Yao, "Optoelectronic oscillators for high speed and high resolution optical sensing," J. Light. Technol., vol. 35, no. 16, pp. 3489-3497, 2017.

[41] X. S. Yao, "Optoelectronic microwave oscillator," J. Opt. Soc. Am. B, vol. 18, pp. 1725-1735, 1996.

[42] X. S. Yao, and L. Maleki, "Multiloop optoelectronic oscillator," IEEE J. Quantum Electron, vol. 36, no. 1, pp. 79-84, 2000.

[43] S. García, and I. Gasulla, "Multi-cavity optoelectronic oscillators using multicore fibers," Opt. Express, vol. 23, no. 3, pp. 2403-2415, 2015.

[44] H. Peng, C. Zhang, X. Xie, T. Sun, P. Guo, X. Zhu, L. Zhu, W. Hu, and Z. Chen, "Tunable DC-60 GHz RF Generation Utilizing a Dual-Loop Optoelectronic Oscillator Based on Stimulated Brillouin Scattering," $J$. Light. Technol., vol. 33, no. 13, pp. 2707-2715, 2015.

[45] B. Haq, S. Kumari, K. Van Gasse, J. Zhang, A. Gocalinska, E. Pelucchi, B. Corbett, and G. Roelkens, "Micro-Transfer-Printed III-V-on-Silicon C-Band Semiconductor Optical Amplifiers," Laser Photonics Rev., vol. 14, no. 7, pp. 1900364, 2020.

[46] L. Chrostowski, X. Wang, J. Flueckiger, Y. Wu, Y. Wang, and S. T. Fard, "Impact of fabrication non-uniformity on chip-scale silicon photonic integrated circuits." in Optical Fiber Communication Conf. San Francisco, CA, USA, 2007, pp. Th2A. 37.

Yue Wang received her bachelor's degree in engineering from the College of Electronic Science and Engineering, Jilin University, Changchun, China, in 2016; she is pursuing her $\mathrm{Ph} . \mathrm{D}$. at the same institution. Currently, she is a visiting Ph.D. student at McGill University working in the area of integrated microwave photonics. Her research interests include optoelectronic oscillators, silicon photonics, microwave photonic filters, and radar and microwave signal processing.

Hao Sun received the B.S. degree in physics from Shandong University in 2015 and the M.Eng. degree from the Institute of Semiconductors, Chinese Academy of Sciences, Beijing, China, in 2018. He is currently pursuing the Ph.D. degree in electrical and computer engineering with McGill University, Montreal, QC, Canada. His research interests include silicon photonics, optical communications, and microwave photonics.

Mostafa Khalil received his B.Sc. in electrical engineering communications and electronics from Alexandria University, Alexandria, Egypt in 2018, and his M.Eng in electrical and computer engineering at McGill University, Montreal, QC, Canada in 2020. He is currently pursuing his Ph.D. in electrical and computer engineering at McGill University, Montreal, QC, Canada. Since his last year at Alexandria University, he has been working on research in the fields of optical communications and silicon photonics.

Wei Dong received her $\mathrm{PhD}$ in microelectronics and solid-state electronics at college of electronic science and engineering, Jilin University, Changchun, China, in 2004. Currently, she is a full professor in the college of electronic science and engineering, Jilin University. Her research interests include microwave photonics, optical fiber sensing and optoelectronic oscillator.
Ivana Gasulla received the M.Sc. degree in telecommunications engineering and the Ph.D. degree in telecommunications from the Universitat Politècnica de València (UPV), Spain, in 2005 and 2008, respectively. She is currently a Senior Researcher (Ramon y Cajal Fellow) and Deputy Director for Dissemination and Promotion at the iTEAM Research Institute of UPV. In 2016, she was awarded a prestigious ERC Consolidator Grant to develop new SpaceDivision Multiplexing technologies for emergent fiber-wireless communications through the project InnoSpace. Her current research interests encompass, among others, the application of multimode and multicore fibers to Microwave Photonics systems. The results of her work have led to more than 100 international publications, highlighting contributions to Nature Communications and Nature Photonics. She is a member of the TPC of the most prestigious conferences in the field: European Conference on Optical Communications (ECOC), Optical Fiber Communication Conference (OFC) and IEEE International Topical meeting on Microwave Photonics (MWP), among others.

José Capmany (S'88-M'91-SM'96-F'08) was born in Madrid, Spain. He received the Ingeniero de Telecomunicacion degree from the Universidad Politécnica de Madrid (UPM), Madrid, Spain, in 1987, the Licenciado en Ciencias F'1sicas degree in 2009, and the Ph.D. degree in electrical engineering and in quantum physics from UPM and the Universidad de Vigo, Vigo, Spain, respectively.

Since 1991, he has been with the Departamento de Comunicaciones, Universidad Politecnica de Valencia (UPV), Valencia, Spain, where he started the activities on optical communications and photonics, founding the Photonics Research Labs Group (www.prl.upv.es). He was an Associate Professor from 1992 to 1995, and since 1996, he has been a Full Professor in photonics and optical communications. He was the Vice-Dean with the Telecommunications Engineering Scholle, UPV, from 1991 to 1996, and the Deputy Head of the Communications Department from 1997 to 2005. From 2005 to 2016, he was the Director with the Research Institute of Telecommunications and Multimedia (iTEAM), UPV (www.iteam.upv.es). He has authored or co-authored more than 550 papers in international refereed journals and conferences. His research activities and interests cover a wide range of subjects related to optical communications including microwave photonics, integrated optics, optical signal processing, fiber Bragg gratings, and more recently quantum cryptography and quantum-photonic information processing.

Dr. Capmany is a Fellow of the Optical Society of America. $\mathrm{He}$ is also a founder and the Chief Innovation Officer of the spin-off company VLC Photonics, Valencia, Spain, (www.vlcphotonics.com) dedicated to the design of photonic integrated circuits and EPHOOX, Valencia, Spain, (www.ephoox.es) dedicated to MWP instrumentation. He was a reviewer for more than $30 \mathrm{SCI}$ journals in the field of photonics and telecommunications. He was a member of the IEEE Photonics Society Board of Governors (2008-2010) and a Distinguished Lecturer for the 2013-2014 term. He was the General Chair for the 41st European Conference on Optical Communications (ECOC), Valencia, Spain, in 2015, and the IEEE International Topical Meeting on Microwave Photonics 
in 2009. He has been a member of the Technical Program Committees of more than 50 international conferences in the field of photonics including ECOC, the Optical Fiber Conference, the Integrated Optics and Optical Communications Conference, CLEO Europe, and the Optoelectronics and Communications Conference. He was an Associate Editor for the IEEE PHOTONICS TECHNOLOGY LeTters (2010-2016) and the IEEE Journal Of LightwaVE TECHNOLOGY (2016-2018). $\mathrm{He}$ has also been a Guest Editor for the IEEE JOURNAL OF SElected TOPICS IN QUANTUM Electronics, the IEEE TRANSACtions ON Microwave TheORY AND TeChNiQues, and Optics Express feature issue on microwave photonics. He is the Editor-in-Chief of the IEEE JOURNAL OF SELECTED Topics IN QuANTUM EleCtronics. He was the 2012 King James I Prize Laureate on novel technologies, the highest scientific distinction in Spain, for his outstanding contributions to the field of microwave photonics and was a recipient of a European Research Council Advanced Grant, in 2016. He is also the recipient of the Extraordinary Doctorate Prize of the Universidad Politecnica de Madrid in 1992.

Lawrence R. Chen (S'95, SM'05) received the B.Eng. degree in electrical engineering and mathematics from McGill University, Montreal, QC, Canada in 1995 and the M.A.Sc. and $\mathrm{Ph} . \mathrm{D}$. degrees in electrical and computer engineering from the University of Toronto, Toronto, ON, Canada in 1997 and 2000, respectively. Since 2000, he has been with the Department of Electrical and Computer Engineering at McGill University. His research interests are in optical communications, silicon photonics, and microwave photonics, as well as engineering education and teaching pedagogy.

Dr. Chen was the Technical Program Chair of the 2019 IEEE International Topical Meeting on Microwave Photonics and has served on technical program committees for a number of international conferences, including the IEEE Photonics Conference, the Conference on Optical Fiber Communications, the Opto-Electronics and Communications Conference, and the Asia Communications and Photonics Conference. He is the Principal Guest Editor of the IEEE Journal OF SELECTED Topics IN QuANTUM ELECTRONICS Issue on Optical Signal Processing (to be published in 2021). He is a Fellow of the Optical Society of America. 